Widefield Science and Technology for the SKA

SKADS CONFERENCE 2009

S.A. Torchinsky, A. van Ardenne, T. van den Brink-Havinga, A.J.J. van Es, A.J. Faulkner (eds.)

4-6 November 2009, Château de Limelette, Belgium

\title{
The Questions that Drive the Specifications
}

\author{
S.A. Torchinsky
}

Station de Radioastronomie de Nançay, 18330 Nançay, France

\begin{abstract}
The fundamental questions to be addressed by the Square Kilometre Array remain relevant today, despite the passage of time since the SKA Science book was published over four years ago. The answer to the questions of Dark Energy, Cosmic Magnetism, Gravitation in Extreme Fields, and the Epoch of Reionisation, all require observational statistics on a very large scale. As a result, the SKA is being designed mainly as a large survey machine. Survey requirements place specific demands on the technical definition of the SKA, and the cost of implementation puts restrictions on the ultimate capability of the SKA. At the same time, we must not trade-off too much of the versatility of the SKA because, as has been seen many times in the past, it is the unexpected discoveries which turn out to be the most important contribution of an instrument.
\end{abstract}

\section{Introduction}

The SKA will play a pivotal role in answering fundamental questions in physics which are currently the focus of the worldwide physics research community. The key science goals of the SKA fit into this global effort to focus on several inter related topics. These include discovering the nature of Dark Energy, the origin of magnetism in the universe, the limits of the General Theory of Relativity, and the formation of the first structure, and the first stars in the Universe. The SKA will also answer questions related to the origin and formation of complex molecules and planetary systems, leading to life on Earth. All these key science goals are described in detail in the SKA Science Book (Carilli \& Rawlings 2004).

The SKADS whitepaper brings these scientific goals into the design of the SKA, and I will review the technical requirements demanded by each science goal, and how it translates into a global vision for the SKA.

The SKADS consortium, primarily through the effort in DS2, has made significant advances in sky simulations, and calibration considerations. This work is accessible to the global SKA community through the SKADS Simulated Skies database, and has already appeared in a number of publications. For example, see Wilman et al. (2008); Obreschkow et al. (2009a,b); Krause et al. (2009); Smits et al. (2009); Santos et al. (2008); Baek et al. (2009). A complete list of SKADS publications can be found on the SKADS website . The SKADS Simulated Skies tools and database are described in more detail by Levrier et al. (2010) and by Klöckner et al. (2010) in these proceedings.

\section{The Cradle of Life}

What conditions are necessary for the development of life?

We are here on the Earth, asking fundamental questions about the nature of the Universe, one of which is how we arrived at such a state that we can do such a thing. The development of

${ }^{\text {a }}$ http://www.skads-eu.org/p/memos.php life on Earth is the result of a number of events in the history of the galaxy and solar system which finally led to the formation of an hospitable environment here on Earth. The questions on which we focus with the SKA are the following:

- How are planetary systems formed?

- Is the solar system a special case?

- How and where are complex (organic) molecules formed?

Once the SKA has its capability at $10 \mathrm{GHz}$ and higher, it will be able to detect the gaps in proto-planetary disks forming around stars. These gaps are where planets are formed (Lazio 2004). It is only recently in the history of astronomy that planets have been detected orbiting other stars. So far, no Earth like planet has been detected. This is almost certainly due to the limitation of current technology, but the possibility remains that Earth is a special case where all the requirements come together to make an environment conducive to the development of life.

Long chain carbon molecules have already been detected in extraterrestrial environments. An example is methanol with a rotational transition emitting at $834 \mathrm{MHz}$. Others are acetaldehyde (at $1.1 \mathrm{GHz}$ ) acetamide (at $9.2 \mathrm{GHz}$ ) and cyclopropenone (at $9.3 \mathrm{GHz}$ ). Further possibilities are listed in Lazio et al. (2009).

\section{Is there anybody out there?}

The radio spectrum has traditionally been the regime where searches have been made for artificially generated signals from civilisations beyond the Earth. Using Earth as an example, there are a few emitters in the radio band, the most powerful of which is the Arecibo planetary radar. The Arecibo S-band radar is used for solar system studies and roughly a dozen times per year it beams a monochromatic, circularly polarised signal (see e.g. Campbell et al. 2003; Black et al. 2007; Carter et al. 2009), with frequency and time domain modulation (Magri et al. 2007) and powers of up to 1 MegaWatt for durations of several hours. For example, during the latter half of 2009, the Arecibo planetary radar operated during 29 sessions of duration averaging 3 hours each, and sometimes for as much as 
10 continuous hours (Hernandez 2009). Such a signal, distinct from any natural phenomenon, could be detected by an SKA type telescope thousands of light years away. If extra terrestrial scientists are studying their planetary system with an Arecibolike instrument, then we could detect their radar, and there are many thousands of stars within the range of detectability of SKA. The advances made in the detection of extra solar planets, and soon the detection of extra solar planets in the habitable zone, make it possible to do long term targeted studies with the SKA.

\section{Tests of Gravity using Pulsars}

\section{How right is Einstein about gravity?}

Observational tests of General Relativity include the demonstration of the existence of gravitational waves (Taylor 1993), and a number of other effects have been observed by timing the orbital characteristics of pulsar-neutron star binary systems. In particular, the double pulsar system (Burgay et al. 2003; Lyne et al. 2004) demonstrates that the theory of General Relativity is correct to $99.95 \%$ (Kramer et al. 2006; Wex \& Kramer 2010). More details of this can be found in these proceedings by Kramer \& Smits (2010) on page 59 and references therein.

What is the strong-field limit at which GR is no longer the correct description of gravity?

Nevertheless, there remains a dichotomy between the quantum theory of physics, and the general theory of relativity. It is expected that at sufficiently large gravitational field strengths, General Relativity will no longer be the correct description of gravity. The SKA will be able to probe this limit by timing a pulsar in orbit around a black hole.

In order to find the most exotic pulsar binary systems which can be used for tests of General Relativity, the SKA will carry out a large survey of the galaxy finding all the observable pulsars, perhaps as many as a few tens of thousands (Smits et al. 2009). Amongst these will be exotic systems needed to carry out the tests.

\section{Is there a gravitational wave background?}

By correlating the measured times-of-arrival of pulses from millisecond pulsars distributed throughout the galaxy, it is possible to infer the passage of a gravitational wave through space where the Earth resides. The times-of-arrival will be slightly early for pulsars in one direction, and later in the opposite direction, with a delay profile that matches what is expected by a gravitational wave. This method of using timing of distributed pulsars is called the Pulsar Timing Array. It is sensitive to gravitational waves of frequency $\mathrm{nHz}$ which is not detectable by any other means.

The SKA may well find a background of gravitational waves and then we can ask: Is it stochastic? That is, is it caused by numerous individual events such as supernovæ and mergers. Or, is it a primordial background arising from the Big Bang itself. In that case, the SKA may be used to probe epochs before the time the Universe cooled sufficiently to release the photons which are the Cosmic Microwave Background. This surface of last scattering is the earliest time which can be probed by observational methods which detect electromagnetic waves. Perhaps the SKA will be able probe times before the surface of last scattering, and enlighten us on the nature of the primordial plasma.

\section{The Epoch of Reionisation}

\section{When was "first light"?}

In the early Universe, after the primordial plasma cooled to allow the creation of neutral material, there followed a long period in which the Universe contained only neutral material, almost entirely neutral hydrogen atoms. The only radiation present was the scattered photons left over from the surface of last scattering, also called the Epoch of Recombination, and observed today as the Cosmic Microwave Background. There was no source of visible light in the Universe during that period which we refer to as the Cosmic Dark Ages. The SKA will probe the Universe for highly redshifted neutral hydrogen gas in order to answer the fundamental questions in the history of the Universe:

- When did the first luminous objects form?

- How did they form, and over what period of time?

The first luminous objects must have emitted large quantities of energetic photons, not only in the visible wavelengths, but also at much higher energies. This radiation then went on to ionise the surrounding neutral medium, creating the largely ionised medium we observe today, which is why that period in the history of the Universe is called the Epoch of Reionisation.

The SKA will carry out a large survey of redshifted neutral hydrogen, giving a history of the evolution of reionisation in the Universe from the time when there was only neutral hydrogen gas to the formation of the first luminous objects. For more details see Zaroubi (2010); Santos et al. (2010) in these proceedings, and, for example, the introduction in Baek et al. (2009).

\section{Cosmic Magnetism}

\section{What is the origin of magnetic fields in the Universe?}

Magnetic fields play a major role in astrophysics at all scales. The formation of stars and planetary systems are intimately connected to the interplay of magnetic fields. Magnetic fields are essential for the protection of life on Earth from energetic charged particles in the solar wind. On galactic scales, magnetic fields are observed to influence, and even determine, the structure of galaxies. See the review in these proceedings on page 93 by Beck (2010).

Despite the obvious importance of magnetic fields to our understanding of the nature of the Universe, we still do not have a strongly supported theory for the origin of magnetic fields.

- Were magnetic fields always there? That is, are they primordial arising from the processes in the early universe? or 
- Were magnetic fields generated over the evolution of the Universe by the dynamo effect in numerous sources distributed throughout the Universe?

The SKA will do an extensive survey of Rotation Measures, recording on the order of 10 million Rotation Measures of sources distributed throughout the sky. This will give statistics on the overall picture of magnetism in the Universe, and we begin to have a handle on possible theories of the origin of magnetic fields. See Beck (2010) in these proceedings on page 93 for more details.

\section{Large Scale Structure}

\section{What is the nature of Dark Energy?}

Observational evidence from distances to super novae combined with statistical analysis on the anisotropies in the Cosmic Microwave Background have shown us that the Universe is undergoing a phase of accelerated expansion (Riess et al. 1998). The more recent analysis of data from the Wilkinson Microwave Anisotropy Probe puts a high precision on this expansion (Komatsu et al. 2009), and allows us to derive conclusions about the energy density of the Universe. The negative energy causing accelerated expansion is called Dark Energy and it appears to account for $\sim 70 \%$ of all energy in the Universe.

The fact that Dark Energy remains an enigma despite its clear importance in the physics of the Universe has led to proposals of numerous experimental projects/ Many of the planned experiments are based on the detection of Baryonic Acoustic Oscillations (Blake \& Glazebrook 2003). Acoustic oscillations in the primordial plasma left a signature on the subsequent formation of structure after the epoch of recombination when the Universe cooled sufficiently to form neutral matter and photons were free to propagate. This signature can be seen in the statistical analysis of the anistropies in the Cosmic Microwave Background. It is also expected to be detected in the distribution of matter in later periods of the Universe.

The SKA will do an all-sky survey measuring simultaneously the position of galaxies, and their redshift by detection of the redshifted $21 \mathrm{~cm}$ forbidden transition of the neutral hydrogen. Over a billion galaxies will be detected, giving the possibility to bin the data and do the analysis in redshift slices. This will improve analysis, and ultimately lead to a high precision determination of the $w$ parameter in the equation of state of the Universe. If $w=1$, then the accelerated expansion of the Universe is entirely explained by the Cosmological Constant in the General Theory of Relativity. We will finally be able to answer the questions:

- Is Dark Energy just a geometrical feature of General Relativity?

- (was Einstein wrong when he thought he was wrong about the Cosmological Constant?)

\section{Transients}

During most of the history of civilisation on Earth, the sky was thought to be mostly static. When viewed with the naked eye, nothing in the heavens appears to change except for the movement of the seven classical planets: The Sun, The Moon, Mercury, Venus, Mars, Jupiter, and Saturn. The appearance of the occasional comet was of the utmost import, because of its rarity.

In more recent times, it has become clear that there are many periodic events in the Universe. Comets are understood to be regular visitors, and the modern era of observational astronomy has discovered transient events on time scales ranging from years, months, days, to subsecond events. Supernovæ explosions have afterglows slowly diminishing over a period of months. Many stars have intrinsic pulsations with periods on the order of days. On the shortest timescales, subpulses of the pulsar in the Crab Nebula have been observed with nanosecond duration (Hankins et al. 2003).

Astronomy is just beginning to get a global view of the various transient populsations, and we can ask the fundamental questions:

- Is there a population of powerful, irregular, transient sources?

- What are they?

- How do they generate the energy they emit?

Also, as discussed above in the section on "Cradle of Life," there is the possibility of artificially generated signals.

The topic of transients is developed in much more detail by Lazio (2010) on page 51 of these proceedings.

\section{Exploration of the Unknown}

\section{Are we asking the right questions?}

The scientific method is a process involving a question followed by studies to achieve an answer. This always leads to further questions and that is the path to discovery. Sometimes however, we can be led astray by the questions we pose. A question may contain a hidden assumption which prevents us from moving towards the correct conclusion.

There have been a number of such examples in the past. One such is the attempt to measure the speed of the Earth with respect to the luminous aether at the end of the 19th century. With everything that was understood about wave propagaion, it seemed unquestionable that electromagnetic waves must propagate through a medium, just as surface waves propagate through water, and pressure waves propagate through air. It was significant step of understanding to imagine that there is no medium through which light propagates.

Perhaps the most famous example in modern scientific history is the question Einstein effectively asked himself before adding a cosmological constant to the General Theory of Relativity. "How can I modify the Theory of General Relativity to conform to the observed, static Universe?" At the time, the Universe appeared to be unchanging. Indeed, the idea of the static, eternal Universe, is deeply rooted in western thinking for nearly 3000 years. Einstein noted that his theory was not stable and added a cosmological constant to counter act the effect of gravity which, on its own, would bring about the collapse of the Universe. He was asking the wrong question. He 
might have asked himself, "What is the theory telling me about the Universe?" and then he could have predicted the expanding Universe given an initial condition of a big bang. Einstein referred to this as his greatest blunder.

In more recent history, telescopes have been built with a specific scientific goal in mind. In most cases, the telescopes become most well known for discoveries and studies completely different from their original science justification. There are a few famous examples.

The 2048 dipole aperture array telescope called the 4-acre array at the Mullard Radio Astrophysical Observatory of the University of Cambridge was built to study scintillation in the interplanetary medium due to the solar wind (Hewish 1974). It discovered pulsars, winning a Nobel prize for Anthony Hewish and Martin Ryle.

A horn antenna used in the 1960's by Bell Labs engineers was originally designed for radio communications with the satellites Echo and Telstar (Wilson 1978). It was afterwards taken over by Bell Labs radio astronomers who wished to make a general survey of cosmic microwave radiation. While doing a precise characterisation of the horn antenna, this led to the disovery of the Cosmic Microwave Background winning a Nobel prize for Penzias \& Wilson.

The $305 \mathrm{~m}$ diameter Arecibo radar was originally conceived by William Gordon to study the ionosphere by measuring RADAR back-scatter from the ionosphere (Cohen 2009). Arecibo is best known for a number of discoveries including the first extra solar planet (Wolszczan 1991), and the long term timing study of the pulsar-neutron star binary system which demonstrated the existence of gravitational waves winning a Nobel prize for Taylor \& Hulse (Taylor 1993).

All these examples show our limited ability to predict the most important results to come from an instrument. Often these discoveries came from the possibility of an instrument to be used in ways that were not considered when it was designed, but the design was sufficiently flexible to allow the instrument to be used in new ways. The design of the SKA must not be entirely optimised for the Key Science projects, and we have to maintain an element of flexibility in the capabilities of the instrument. This is where the most important discoveries will arise. It is the flexibility of the instrument, and the imagination of the next generation of astronomers to think of new ways to use the instrument, and to come up with the right questions to ask.

\section{Specifications Overview}

The science goals summarised in the last section cover various topics, but have in common the desire to understand fundamental aspects of physics and the nature of the universe. As a result, the required observations are necessarily global in nature. That is, in order to reach conclusions about the global properties of the universe, one must observe as much of the universe as possible. The result is a requirement to conduct large scale surveys.

Surveys serve two main functions. In one case, the statistics of the survey itself is the primary interest. This is the case for example for the Baryonic Acoustic Oscillations experiment, and the all-sky rotation-measure survey. In the second case, the survey serves as a tool to discover extreme objects. This is the case for the pulsar survey looking for exotic binary systems to test gravity, or looking for isolated pulsars to use in the Pulsar Timing Array, as is the search for new classes of transient sources.

Surveys with the goal of discovering new objects require follow-up observations of the newly discovered objects. Such observations will often demand high angular resolution, pushing out the SKA baseline length requirement.

As part of the SKADS Virtual Telescope exercise, the instrument capabilities required by the SKA have been summarised in Table 1. The Epoch of Reionisation experiment and the Baryonic Acoustic Oscillations experiment are both well served by aperture array technology. A sparse array similar to LOFAR is required for the EoR experiment, while a dense array for the mid frequencies is necessary for the BAO experiment, providing the large field of view and subsequent mapping speed which is unequalled by any other technology. Other requirements include polarisation purity, necessary for the Cosmic Magnetism key project. The dynamic range cited here assumes a relatively quiet site in terms of Radio Frequency Interference, and depends more on the requirement of large field of view imaging. The longest baselines are necessary for follow-up ob servations such as VLBI of pulsars, detailed studies of new discoveries. Long baselines are particularly important when SKA $\Omega$ Phase-D is online with its frequencies up to $10 \mathrm{GHz}$ and then the studies of protoplanetary disks can begin, as well as study of organic molecules in planet forming systems, and especially. in solar system analogs. In general, however, it is the large surveys which drive the specifications, and these require more collecting area in the core rather than at long baselines.

\section{Summary}

The design of the Square Kilometre Array is motivated by a number of fundamental questions in physics and astronomy. These have been grouped together in five main topics.

For "The Cradle of Life" we ask "What conditions are necessary for the development of life?" leading us to build an instrument capable of detecting complex molecules, and detecting the early stages of planetary formatin in stellar systems.

For "Tests of Gravity using Pulsars" we ask "How right was Einstein about gravity?" which requires an instrument capable of finding all the detectable pulsars in the galaxy and timing many of them in order to test the theory of General Relativity in extreme gravity fields, and in order to detect nanoHertz gravitational waves.

For "The Epoch of Reionisation" we ask "When was first light?". This requires a low frequency instrument capable of surveying the whole sky with great sensitivity in order to measure the redshifted neutral hydrogen at different epochs in the early Universe.

For "Cosmic Magnetism" we ask "What is the origin of magnetic fields in the Universe?" In order to answer this question the SKA must be capable of doing a large survey of over 10 million Rotation Measures. This puts strict constraints on the imaging capability and polarisation purity of the instrument. 
For "Large Scale Structure" we ask "What is the nature of Dark Energy?" This leads to the requirement that we build an instrument capable of measuring the signature of the Baryonic Acoustic Oscillations in the distribution of matter in the Universe. This is to be done by a large survey of over a billion galaxies measuring simulataneously their positions and redshift. The SKA thus requires a very large field of view combined with high fidelity imaging and spectroscopic capabilities necessary to measure the weak signal of redshifted neutral hydrogen.

These are the original five Key Science topics on which the SKA focuses, but there has been more and more emphasis on the topic of the transient Universe where we ask "Is there a population of powerful, irregular, transient sources, and what is their nature?" The SKA will require not only the capability to measure events on short time scales, but to do so over a very wide field of view in order to have a better likelihood to catch those transient events, wherever they may occur.

All these fundamental questions arise from our current understanding of the Universe, but we could well be asking the wrong questions. Many past instruments have become famous for discoveries and for long term projects that were not imagined by the builders of those instruments. The SKA needs to have capabilities allowing it to make discoveries that we have not imagined. It will be used in ways that were not yet considered, by people who are now only school age, and by people not yet born. The SKA design must include capabilities which go beyond those strictly required by the key science topics outlined here and include an element of flexibility which will permit those future astronomers to make the discoveries that we have not imagined.

Acknowledgements. During the course of the SKADS Project, the author was jointly funded by the Observatoire de Paris, by the Conseil national de la recherche scientifique (CNRS) and by the European Commission Framework Programme 6, Square Kilometre Array Design Studies (SKADS), contract no. 011938.

\section{References}

Baek, S., Di Matteo, P., Semelin, B., Combes, F., \& Revaz, Y. 2009, A\&A, 495, 389

Beck, R. "Wide Field Polarimetry and Cosmic Magnetism," 2010, in Proc. Wide Field Science and Technology for the SKA, Limelette, Belgium, S.A. Torchinsky et al. (eds), ASTRON, ISBN 978-90-805434-5-4

Black, G.J., Campbell, D.B., \& Carter, L.M. 2007, Icarus, 191, 702

Blake, C., \& Glazebrook, K. 2003, ApJ, 594, 665

Burgay, M., et al. 2003, Nature, 426, 531

Campbell, D.B., Black, G.J., Carter, L.M., \& Ostro, S.J. 2003, Science, 302, 431

Carilli, C., \& Rawlings, S. (eds) 2004, Elsevier

Carter, L. M., Campbell, B. A., Hawke, B. R., Campbell, D. B., \& Nolan, M. C. 2009, Journal of Geophysical Research (Planets), 114, 11004

Cohen, M.H. 2009, J. Astronomical History and Heritage, 12, 2, 141-152
Hankins, T. H., Kern, J. S., Weatherall, J. C., \& Eilek, J. A. 2003, Nature, 422, 141

Hernandez, H., 2009, The Arecibo schedule, available at http: //www . naic.edu/vscience/schedule/ scedfra2.htm

Hewish, A., Nobel lecture, 1974, The Nobel Foundation http://nobelprize.org/nobel_prizes/physics/ laureates/1978/wilson-lecture.pdf

IEEE Puerto Rico \& Caribean section, 2001, IEEE Global History Network http://www.ieeghn. org/wiki/index . php?title=Milestones : NAIC/ Arecibo_Radiotelescope $\backslash \% 2 C \_1963 \backslash \& o l d i d=38928$

Klöckner, H.R., Auld, R., Heywood, I., Obreschkow, D., Levrier, F., Rawlings, S. "SKA HI end2end simulation," 2010, in Proc. Wide Field Science and Technology for the SKA, Limelette, Belgium, S.A. Torchinsky et al. (eds), 2 ASTRON, ISBN 978-90-805434-5-4

Komatsu, E., et al. 2009, ApJS, 180, 330

Kramer, M., et al. 2006, Science, 314, 97

Kramer, M., Smits, R., "Pulsars - Enabling the gravita $\uparrow$ tion KSP of the SKA," 2010, in Proc. Wide Field Science and Technology for the SKA, Limelette, Belgium, S.A. Torchinsky et al. (eds), ASTRON, ISBN 978-90805434-5-4

Krause, M., Alexander, P., Bolton, R., Geisbüsch, J., Green, 2 D. A., \& Riley, J. 2009, MNRAS, 400, 646

Lazio, T.J.W. in "Science with the Square Kilometre Array" C. Carilla \& S. Rawlings (eds) 2004, Elsevier

Lazio, T.J.W. and the SKA Science Working Group, "The Design Reference Mission", SKA Program Development Office, http://www.skatelescope. org/PDF/091001_DRM_vQ.4.pdf

Lazio T.J.W., "Radio Transients and Aperture Arrays," 2010, in Proc. Wide Field Science and Technology for the SKA, Limelette, Belgium, S.A. Torchinsky et al. (eds), ASTRON, ISBN 978-90-805434-5-4

Levrier, F., Wilman, R.J., Obreschkow, D., Klöckner, H.-R., Heywood, I., Rawlings, S., "Mapping the SKA Simulated Skies with the $S^{3}$-Tools," 2010, in Proc. Wide Field Science and Technology for the SKA, Limelette, Belgium, S.A. Torchinsky et al. (eds), ASTRON, ISBN 978-90805434-5-4

Lyne, A. G., et al. 2004, Science, 303, 1153

Magri, C., Ostro, S. J., Scheeres, D. J., Nolan, M. C., Giorgini, J. D., Benner, L. A. M., \& Margot, J.-L. 2007, Icarus, 186, 152

Obreschkow, D., Klöckner, H.-R., Heywood, I., Levrier, F., \& Rawlings, S. 2009, ApJ, 703, 1890

Obreschkow, D., Croton, D., D eLucia, G., Khochfar, S., \& Rawlings, S. 2009, ApJ, 698, 1467

Riess, A. G., et al. 1998, AJ, 116, 1009

Santos, M. G., Amblard, A., Pritchard, J., Trac, H., Cen, R., \& Cooray, A. 2008, ApJ, 689, 1

Santos, M.G., Ferramacho, L., Silva, M.B., "Fast Large Volume Simulations of the Epoch of Reionization," 2010, in Proc. Wide Field Science and Technology for the SKA, Limelette, Belgium, S.A. Torchinsky et al. (eds), ASTRON, ISBN 97890-805434-5-4 
Table 1: Instrument requirements for the SKA over its construction stages, determined using the scientific requirements and costing exercise done within the SKADS project.

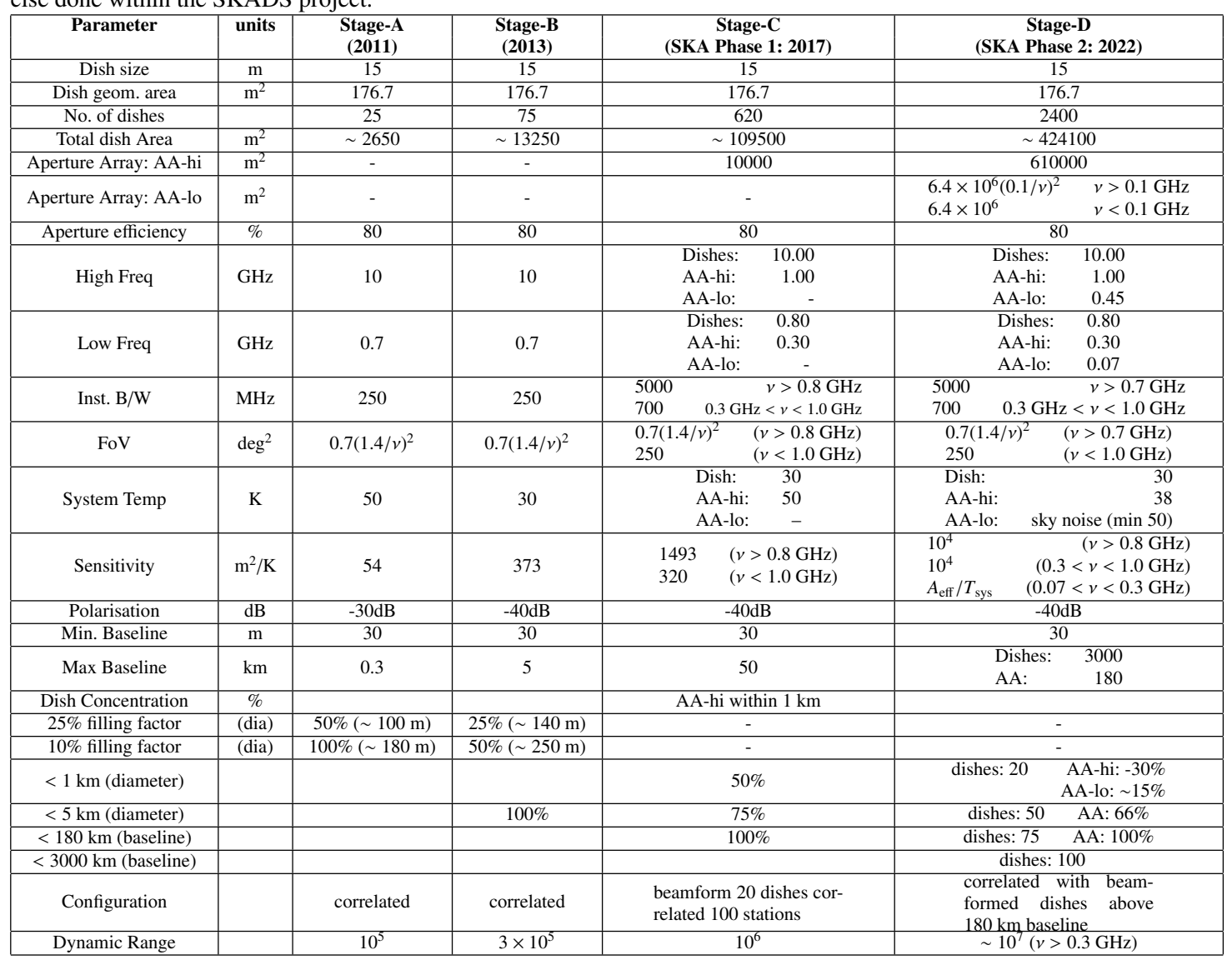

Smits, R., Kramer, M., Stappers, B., Lorimer, D. R., Cordes, J., \& Faulkner, A. 2009, A\&A, 493, 1161

Taylor, J.H., Nobel lecture, 1993, The Nobel Foundation http://nobelprize.org/nobel_prizes/physics/ laureates/1993/taylor-lecture.pdf

Wex, N., \& Kramer, M. 2010, arXiv:1001.4733

Wilman, R. J., et al. 2008, MNRAS, 388, 1335

Wilson, R.W., Nobel lecture, 1978, The Nobel Foundation http://nobelprize.org/nobel_prizes/physics/ laureates/1978/wilson-lecture.pdf

Wolszczan, A. 1991, BAAS, 23, 1347

Zaroubi, S., "Probing the Epoch of Reionization with Low Frequency Arrays," 2010, in Proc. Wide Field Science and Technology for the SKA, Limelette, Belgium, S.A. Torchinsky et al. (eds), ASTRON, ISBN 978-90805434-5-4 